\title{
Les modalités de l'excrétion de l'iode radioactif dans le lait des ruminants
}

\author{
par \\ F. DABURON, J. ROMAIN, Y. TRICAUD \\ et P. NIZZA*
}

\section{I. - POSITION DU PROBLEME}

Le lait est la source la plus importante d'iode radioactit cans la contamination de la chaîne alimentaire. En effet, la contamination des plantes par les retombées atmosphériques est essentiellement directe sur les feuilles, et les conditions d'alimentation des animaux dans les prés, broutant de larges surfaces, sont éminemment favorables à l'absorption de quantités notables d'iode (presque exclusivement, l'iode 131) [7].

\section{II. - LES BILANS D'EXCRETION}

Ils sont établis en général dans deux conditions d'administration : administration unique ou administration chronique. En fait, l'étude la plus valable, du point de vue de la protection sanitaire, devrait être réalisée en administration chronique avec des doses journalières décroissantes selon une période de $5 \mathrm{j}$ : ce chiffre est la résultante de la décroissance physique de l'iode $131(8 \mathrm{j})$ et de ce que les anglo-saxons appellent le "field loss factor ", c'est-à-dire, la décontamination naturelle due à des facteurs physiques et chimiques, qui a une période de 14 jours environ sous nos climats.

La figure 1 donne les courbes d'excrétion dans le lait chez la vache, selon les trois modalités de contaminations décrites ci-dessus.

a) En ce qui concerne les administrations uniques, nous avons obtenu les chiffres suivants :

1) Chez LA BREBIS : (13 animaux, de 1962 à 1967) une moyenne de 35 p. 100 de la dose ingérée excrétée par le lait (30 p. 100 par

* Laboratoire-Etable de Radiobiologie Appliquée. (D.P.S. - Commissariat à l'Energie Atomique) (78) Jouy-en-Josas. 
TABLEAU 4. - Pourcentage d'iode $131 \mathrm{du}$ lait fixé sur résine anionique après contamination unique (brebis)

\begin{tabular}{|c|c|c|c|c|c|c|c|c|c|c|c|}
\hline $\begin{array}{l}\text { Contamination : } \\
\qquad 8 \mathrm{~h} \mathrm{j} 0\end{array}$ & В $13-1$ & B 14 & B 15 & B 33 & B 27 & B $13-2$ & B 22 & B 25 & B 31 & $\begin{array}{c}\overline{\mathrm{m}} \\
\text { en p. } 100\end{array}$ & $\sigma$ \\
\hline $17 \mathrm{~h} \mathrm{j} 0$ & 97,9 & 98,8 & 99,3 & 93,5 & & & 93,6 & & & 97,2 & 2,1 \\
\hline $\begin{array}{rl}8 \mathrm{~h} & \\
17 \mathrm{~h} & \mathrm{j} 1\end{array}$ & $\begin{array}{l}95,3 \\
94,7\end{array}$ & $\begin{array}{l}97,2 \\
96,3\end{array}$ & $\begin{array}{l}99,3 \\
98,5\end{array}$ & $\begin{array}{l}88,3 \\
85,5\end{array}$ & 88,4 & & $\begin{array}{l}97,1 \\
93,8\end{array}$ & 88,3 & 76,4 & $\begin{array}{l}91,3 \\
93,8\end{array}$ & $\begin{array}{l}7,1 \\
4,4\end{array}$ \\
\hline $\begin{array}{r}8 \mathrm{~h} \\
17 \mathrm{~h}\end{array} \quad \mathrm{j} 2$ & $\begin{array}{l}94,8 \\
92,1\end{array}$ & $\begin{array}{l}96,3 \\
95,5\end{array}$ & $\begin{array}{l}98,7 \\
98,5\end{array}$ & $\begin{array}{l}84,4 \\
86,4\end{array}$ & 93,2 & & $\begin{array}{l}91,3 \\
90,5\end{array}$ & 84,4 & 84,3 & $\begin{array}{l}90,9 \\
92,6\end{array}$ & $\begin{array}{l}5,5 \\
4,1\end{array}$ \\
\hline $\begin{array}{r}8 \mathrm{~h} \\
17 \mathrm{~h}\end{array}$ j 3 & $\begin{array}{l}89,3 \\
86,3\end{array}$ & $\begin{array}{l}95,9 \\
96,1\end{array}$ & $\begin{array}{l}98,5 \\
98,2\end{array}$ & 88,9 & 77,9 & 92,9 & 91,4 & 80,7 & 84,6 & $\begin{array}{l}88,9 \\
93,5\end{array}$ & $\begin{array}{l}6,4 \\
5,2\end{array}$ \\
\hline $\begin{array}{rl}8 \mathrm{~h} & \mathrm{j} 4\end{array}$ & $\begin{array}{l}71,9 \\
59,5\end{array}$ & $\begin{array}{l}96,5 \\
96,5\end{array}$ & $\begin{array}{l}98,2 \\
98,4\end{array}$ & & 87,9 & 93,4 & 92,1 & 77,1 & 85,4 & $\begin{array}{l}87,8 \\
84,8\end{array}$ & $\begin{array}{r}8,7 \\
17,9\end{array}$ \\
\hline $\begin{array}{cc}8 \mathrm{~h} & \\
17 \mathrm{~h} & \mathrm{j} 5\end{array}$ & $\begin{array}{l}40,2 \\
56,1\end{array}$ & $\begin{array}{l}96,7 \\
94,6\end{array}$ & 97,2 & & 85,2 & 95,7 & & 71,8 & 86,3 & $\begin{array}{l}81,9 \\
75,3\end{array}$ & $\begin{array}{l}18,9 \\
19,2\end{array}$ \\
\hline $\begin{array}{rl}8 \mathrm{~h} & \\
17 \mathrm{~h} & \mathrm{j} 6\end{array}$ & $\begin{array}{l}57,9 \\
56,2\end{array}$ & $\begin{array}{l}94,9 \\
95,9\end{array}$ & & & 87,4 & 96,9 & $\begin{array}{l}90,3 \\
79,8\end{array}$ & 83,6 & 86,2 & $\begin{array}{l}85,3 \\
77,3\end{array}$ & $\begin{array}{l}12,0 \\
16,3\end{array}$ \\
\hline $\begin{array}{rl}8 \mathrm{~h} & \\
17 \mathrm{~h} & \mathrm{j} 7\end{array}$ & 65,9 & $\begin{array}{l}95,5 \\
91,7\end{array}$ & $\begin{array}{l}93,6 \\
92,9\end{array}$ & & 80,0 & 97,1 & $\begin{array}{l}84,7 \\
89,4\end{array}$ & 87,7 & 87,4 & $\begin{array}{l}86,5 \\
91,3\end{array}$ & $\begin{array}{l}9,5 \\
1,6\end{array}$ \\
\hline $\begin{array}{r}8 \mathrm{~h} \\
17 \mathrm{~h}\end{array} \quad \mathrm{j} 8$ & & $\begin{array}{l}93,3 \\
64,5\end{array}$ & $\begin{array}{l}93,0 \\
93,2\end{array}$ & & 92,5 & 96,9 & $\begin{array}{l}89,0 \\
82,8\end{array}$ & 89,4 & 90,0 & $\begin{array}{l}92,0 \\
80,2\end{array}$ & $\begin{array}{r}2,7 \\
11,9\end{array}$ \\
\hline $\begin{array}{r}8 \mathrm{~h} \\
17 \mathrm{~h}\end{array}$ & & $\begin{array}{l}88,6 \\
84,0\end{array}$ & $\begin{array}{l}91,9 \\
94,1\end{array}$ & & 83,3 & 96,4 & $\begin{array}{l}93,0 \\
91,6\end{array}$ & 88,5 & 89,2 & $\begin{array}{l}90,1 \\
89,9\end{array}$ & $\begin{array}{l}4,0 \\
4,3\end{array}$ \\
\hline $\begin{array}{r}8 \mathrm{~h} \\
17 \mathrm{~h}\end{array}$ & 56,2 & $\begin{array}{l}88,9 \\
55,3\end{array}$ & $\begin{array}{l}94,6 \\
95,9\end{array}$ & & 73,1 & 96,7 & $\begin{array}{l}92,5 \\
89,3\end{array}$ & 87,0 & 89,9 & $\begin{array}{l}84,4 \\
80,1\end{array}$ & $\begin{array}{l}13,8 \\
17,8\end{array}$ \\
\hline $\begin{array}{rl}8 \mathrm{~h} & \\
17 \mathrm{~h} & 11 \mathrm{j}\end{array}$ & 51,4 & $\begin{array}{r}83,4 \\
83,7\end{array}$ & $\begin{array}{l}96,1 \\
97,1\end{array}$ & & 80,1 & 97,0 & 90,0 & 84,5 & 85,9 & $\begin{array}{l}81,7 \\
90,4\end{array}$ & $\begin{array}{r}13,3 \\
6,7\end{array}$ \\
\hline $8 \mathrm{~h}$ & & 90,4 & 96,1 & & 80,1 & 97,9 & 75,6 & 72,3 & 82,6 & 85,0 & 9,2 \\
\hline
\end{tabular}





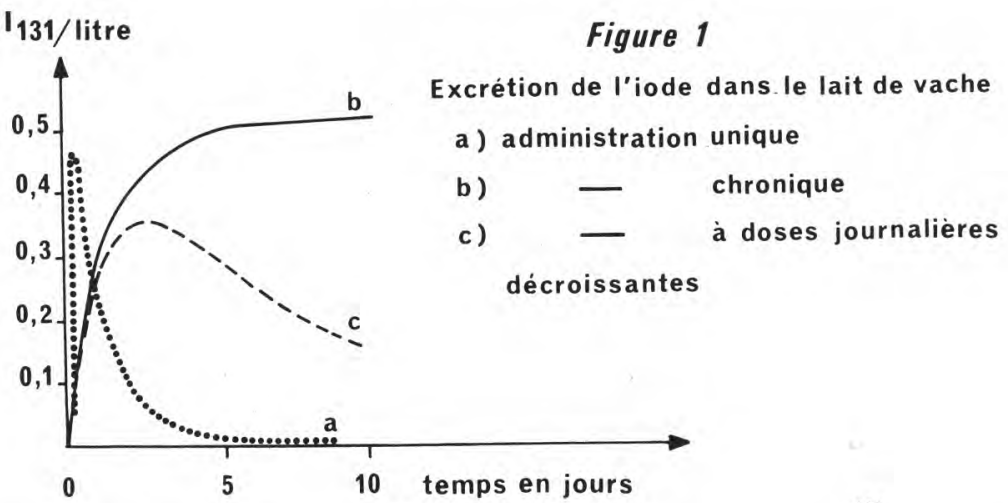

(1)

l'urine - 15 p. 100 dans les fécès), les chiffres extrêmes étant 18 et 65 p. 100, pour une période de prélèvement de $15 \mathrm{j}$ environ [5-6].

La quantité d'iode éliminé varie dans le même sens que la quantité de lait produite mais le coefficient de régression entre les deux n'est pas significatif (calcul effectué avec 18 animaux).

2) Chez la vache :

En février, 16 p. 100 (pour 14 l/j) avec un maximum de fixation dans la thyroïde de 24 p. 100 le $4^{\text {me }}$ jour.

En juin, 7 p. 100 (pour 7 l/j) ; 16,3 p. 100 (pour 22,3 l/j) avec un maximum de fixation dans la thyroïde de 20 p. 100 le $5^{\text {me }}$ jour.

La concentration est maximale dans le lait (de 0,4 à 0,5 p. 100 de la dose administrée par litre) entre 10 et $18 \mathrm{~h}$.

L'excrétion journalière se présente selon deux exponentielles de périodes approximatives 1 et $5 \mathrm{j}$. La période de la seconde exponentielle est à rapprocher de la période effective de la thyroïde (fig. 2).

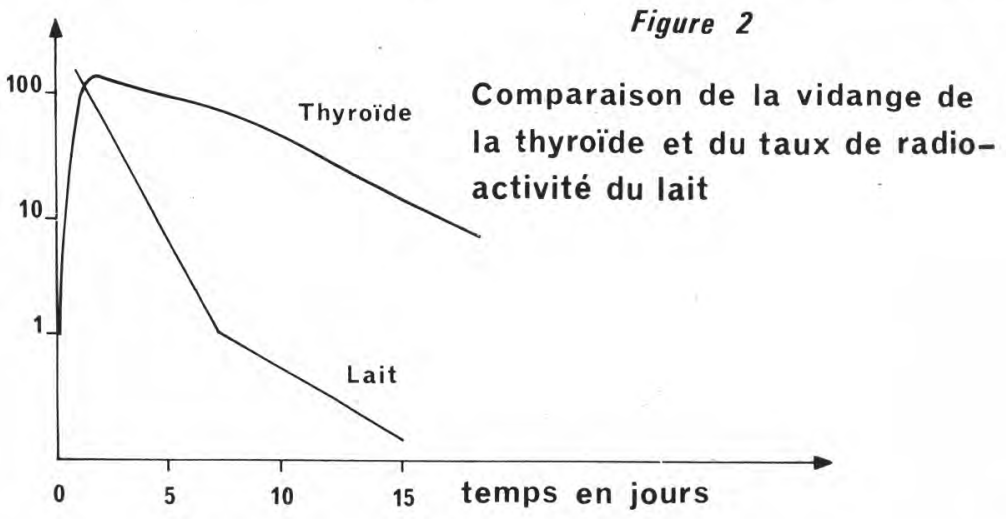

(1) D'après réf. 7, fig. 14.2, p. 303. 


\section{b) En administration chronique, nous trouvons :}

CHEZ UNE BREBIS :

- 40 p. 100 de la dose totale dans le lait,

- 15 p. 100 dans les fécès.

- 40 p. 100 dans l'urine,

La contamination avait duré $10 \mathrm{j}$ : la concentration moyenne est de 10 p. 100 de la dose journalière par litre de lait.

Chez 4 vaches recevant $10 \mu \mathrm{Ci} / \mathrm{j}$, pendant 1 mois :

\begin{tabular}{|c|c|c|c|}
\hline & $\begin{array}{l}\text { Q totale } \\
\text { excrétée } \\
\text { (p. 100) }\end{array}$ & $\begin{array}{c}\text { Q de lait } \\
\text { journalier } \\
\text { (1) }\end{array}$ & $\begin{array}{c}\text { p. } 100 \text { de la } \\
\text { dose } \\
\text { quotidienne } / 1\end{array}$ \\
\hline En début de lactation : & & & \\
\hline 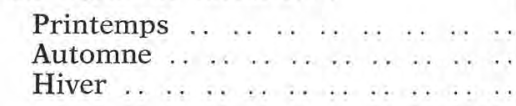 & $\begin{array}{c}7 \\
3 \\
15,5\end{array}$ & $\begin{array}{l}12 \\
11 \\
17,5\end{array}$ & $\begin{array}{l}0,75 \\
0,35 \\
1,25\end{array}$ \\
\hline $\begin{array}{l}\text { En fin de lactation : } \\
\text { Eté } \ldots . . \\
\end{array}$ & 3 & 8,6 & 0,5 \\
\hline
\end{tabular}

L'activité spécifique du lait devient constante 8 à $10 \mathrm{j}$ après le début de la contamination chronique. Après arrêt de l'administration quotidienne, l'activité spécifique du lait décroît avec une période de $1 \mathrm{j}$ environ pendant les 5 à $6 \mathrm{j}$ où nous avons pu la suivre.

Tous ces chiffres sont en accord avec ceux donnés par les auteurs anglo-saxons [7-13-16-27].

\section{III. - FACTEURS INFLUANT SUR LE TRANSFERT DE L'IODE DU SANG AU LAIT}

\section{a) Forme chimique sous laquelle l'iode est ingéré}

- Dans les retombées atmosphériques, l'iode radioactif est à $80-85$ p. 100 sous forme $\mathrm{I}^{-}, \mathrm{I}_{2}$ ou $\mathrm{I}$ organique et à $15-20$ p. 100 sous forme $\mathrm{IO}^{-}{ }_{3}$ [7]. Du point de vue de la cinétique de l'iode, les formes iodates de sodium et de calcium sont identiques à la forme iodure [15].

- L'iode lié aux protéines du lait, administré au veau, présente la particularité d'être excrété deux fois plus que l'iodure dans les urines et d'être capté deux fois moins par la thyroïde [29].

Chez la chèvre, on retrouve dans le lait 15 p. 100 de l'iode administré per os sous forme d'iodocaséine et 50 p. 100 de l'iode administré sous forme iodure [31]. 


\section{b) Certains produits agissant sur le métabolisme de l'iode dans la thyroide peuvent avoir une action sur la mamelle}

- Chez la chèvre, un excès d'iodure stable $(1,2 \mathrm{~g} / \mathrm{j})$ diminue l'excrétion du radioiode [12-16] lors d'une administration continue, alors qu'une faible dose $(4 \mathrm{mg} / \mathrm{j})$ l'augmente.

Lors d'une tentative de décontamination, après ingestion d'une dose unique, par injection massive d'iodure stable [6], on constate le même phénomène. Chez la brebis, l'élimination par l'urine augmente de façon notable tandis que l'excrétion par le lait diminue. La première exponentielle de la courbe d'excrétion journalière prend une part prépondérante ; le changement de pente a lieu entre le $8^{\mathrm{m} \theta}$ et le $11^{\text {me }} \mathrm{j}$ au lieu de 5 à $7 \mathrm{j}$ pour les animaux témoins (fig. 2 ).

Les chlorates, les perchlorates, les fluoroborates et les thiocyanates qui bloquent la captation de l'iodure dans la thyroïde entraînent une diminution de la quantité d'iode du lait [12]. Par contre, le thiouracile qui bloque l'oxydation de l'iodure en iode élémentaire (qui seul peut halogéner les groupements tyrosiniques et la thyroglobuline) n'a pas d'action sur l'excrétion de l'iode par le lait [7-13-19-25]. Pourtant, la thiourée à la dose de $1 \mathrm{~g} / \mathrm{j}$, abaisserait de façon notable le pourcentage éliminé par le lait chez la chèvre [12].

- La thyroxine, injectée par voie intraveineuse réduit la quantité d'iode excrété par la mamelle : elle semble diminuer le pouvoir de concentration de la glande en diminuant le rapport $\mathrm{R}$ :

$$
\mathrm{R}=\frac{\text { Iodure du lait }}{\text { Iodure du plasma }}
$$

Dix mg de $\mathrm{L}_{-} \mathrm{T}_{4}$, administrés quotidiennement à 4 vaches entrâ̂nent une baisse du rapport $\mathrm{R}$ et de la concentration dans le lait d'un facteur 2 [13]. A propos de cette étude, Lengemann donne les valeurs normales des rapports $\mathrm{R}$ déterminés chez la vache d'une part, chez la brebis et la chèvre, d'autre part, soit $0,6<R<5,5 \mathrm{chez}$ la vache et $2<\mathrm{R}<12 \mathrm{chez}$ la chèvre. On peut tenter d'expliquer ainsi les différences très importantes entre les quantités excrétées par le lait chez ces deux espèces. Les valeurs de ces rapports supérieures à l'unité, peuvent faire penser à un processus actif pour l'excrétion de l'iode dans le lait. Reineke [25] a trouvé une corrélation étroite entre la quantité d'iode excrétée par le lait et la valeur du rapport $\mathrm{R}$; la valeur de ce rapport donnerait une bonne appréciation de l'activité de la glande, qui retentit évidemment sur la production laitière.

La thyroxine semble toutefois avoir deux actions qui s'opposent du point de vue de l'excrétion de l'iode dans le lait : Miller [21] en faisant ingérer $80 \mathrm{mg}$ de L-Thyroxine par jour à des vaches laitières, observe une augmentation de la production laitière et de l'excrétion d'iode par le lait, sans augmentation de la concentration. 
On peut penser en conclusion que selon les doses, les états physiologique des animaux et leur taux de production laitière, la thyroxine augmente l'excrétion d'iode en augmentant la quantité de lait produite et la réduit en diminuant le rapport des concentrations dans le lait et le sang. Le résultat dépend de la réponse maximale des individus dans un sens ou dans un autre.

\section{c) L'état physiologique de la thyroïde}

\section{EFFET DE LA SAISON}

L'activité thyroïdienne est maximale au début de l'hiver et minimale au printemps [5-7]. On peut s'attendre avec Lengemann, qu'au minimum d'activité dans la thyroïde corresponde le maximum d'activité dans le lait et réciproquement [7-16]. Nous avons vu, ci-dessus, l'effet de la thyroxine et nous connaissons la compétition qui existe dans l'organisme entre la thyroïde, le rein et la mamelle vis-à-vis de la captation de l'iode. Nous n'avons pas pu comme Garner [7] mettre en évidence ce phénomène ni en administration unique, ni en contamination chronique, pas plus chez les brebis que chez les vaches [6].

Il est possible que cette influence de la saison soit relativement difficile à faire apparaître avec des animaux placés en expérimentation dans les locaux maintenus à température constante et nourris avec un régime uniforme. Pourtant, on remarque nettement, dans la majorité des cas, les différences d'activité de la thyroïde. Peut-être le rôle de l'alimentation permettrait d'expliquer cette opposition dans les résultats obtenus.

\section{EFFET D'UNE ADMINISTRATION PRÉALABLE D'IODE RADIOACTIF [19-20]}

Miller et al. ont administré à 7 vaches entre 140 et $210 \mu \mathrm{Ci} / \mathrm{kg}$ de poids vif d'iode 131, correspondant à des doses comprises entre 12000 et $17000 \mathrm{rad}$ pour la thyroïde. Chaque animal était apparié avec sa jumelle qui ne recevait pas d'iode et servait de témoin. On constate 2 ans après que :

- La production laitière pour une lactation est chez l'animal irradié égale à 50 p. 100 de celle du témoin (elle est de 70 p. 100 au début et baisse plus rapidement).

- La fixation thyroïdienne est de 3 p. 100 à $72 \mathrm{~h}$ au lieu de 17 p. 100.

- Le taux d'iodure plasmatique est 2 à 3 fois plus élevé, le rapport iodure du lait / iodure du plasma est 2 fois plus élevé chez l'irradié.

L'excrétion par le lait est 2 fois plus importante (13 p. 100 au lieu de 7 p. 100 de la quantité ingérée) bien que la production de lait soit plus faible. Ceci peut être dû :

- à une compétition plus faible de la thyroïde, 
- à l'existence de formes circulantes anormales passant plus facilement dans la mamelle.

On peut aussi voir dans ce phénomème une preuve de l'indépendance entre l'excrétion du lait et celle de l'iode, mais à ces doses, on peut penser que la mamelle est lésée. Cependant, l'injection de thyroxine remonte le taux des P.B.I. à la normale et abaisse le taux d'excrétion lactée.

\section{d) Intervalle entre les traites [18]}

Lorsqu'on trait chez une même vache, deux quartiers d'un côté toutes les $8 \mathrm{~h}$ et les deux quartiers de l'autre côté toutes les $32 \mathrm{~h}$ on note, en $6 \mathrm{j}$, une excrétion de 40 p. 100 supérieure du côté en rétention, bien que la quantité de lait produite soit de 35 p. 100 inférieure à celle du côté trait toutes les $8 \mathrm{~h}$. L'excrétion maximale a lieu pendant les 32 premières heures. Le passages de ${ }^{131} \mathrm{I}$ serait donc augmenté avec la réplétion de la mamelle. Nous avons cependant observé le phénomène inverse chez la brebis.

\section{IV. - BIOCHIMIE DE L'EXCRETION}

On sait depuis un certain temps [7-18-22-30] que l'iode du lait existe sous deux formes : l'iode ionique et l'iode liée aux protéines. Jeanmaire [8] a montré le premier que le pourcentage d'iode lié augmentait en fonction du temps, après une administration unique chez une vache. Nous avons repris systématiquement cette étude avec 9 brebis et 3 vaches en contamination unique et 2 vaches en contamination chronique en passant le lait excrété quotidiennement sur colonnes de résine anionique (Dowex $1 \times 8$ ), selon la technique décrite par Jeanmaire [8].

On note (fig. 3) que le pourcentage fixé sur colonne, c'est-à-dire l'iode ionique, diminue de 95 à 85 p. 100 en $6 \mathrm{j}$ chez la brebis et de 95 à 65 p. 100 en $8 \mathrm{j}$ chez la vache (courbes moyennes avec 9 brebis et 3 vaches). A l'équilibre, les chiffres varient très irrégulièrement : entre 75 et 90 p. 100 pour la brebis, entre 55 et 85 p. 100 pour les vaches (tab. 4).

Il est intéressant de comparer pendant le même temps, l'évolution des pourcentages iodures et "iode organique » (P.B.I.) du sérum. Chez la brebis comme chez la vache, le pourcentage ionique passe de 95 à 10 p. 100 en 6 à 8 j.

Lors d'une administration chronique, le pourcentage ionique dans le lait varie entre 90 et 80 p. 100 tandis que dans le sérum, le pourcentage d'iodure est relativement constant et égal à 70 p. 100 [6].

Il est à noter également que dans le cas d'un blocage de la thyroïde par un excès d'iode stable - sous forme ionique (Naiodine ND) et organique lentement résorbable (Lipiodol ND) - injecté 3 , 
6 et $13 \mathrm{~h}$ après ingestion de ${ }^{131} \mathrm{I}$ chez 3 brebis, le pourcentage d'iode ionique reste constant dans le lait et égal à $98-100$ p. 100 pendant les $10 \mathrm{j}$ que durait l'expérimentation. A ce moment, pourtant, le taux de P.B.I. du sérum obtenu par précipitation à l'acide trichloracétique est de 86 p. 100 pour les brebis décontaminées à 6 et $13 \mathrm{~h}$, 59 p. 100 pour celle injectée à $3 \mathrm{~h}$ et 93 p. 100 pour le témoin [6].

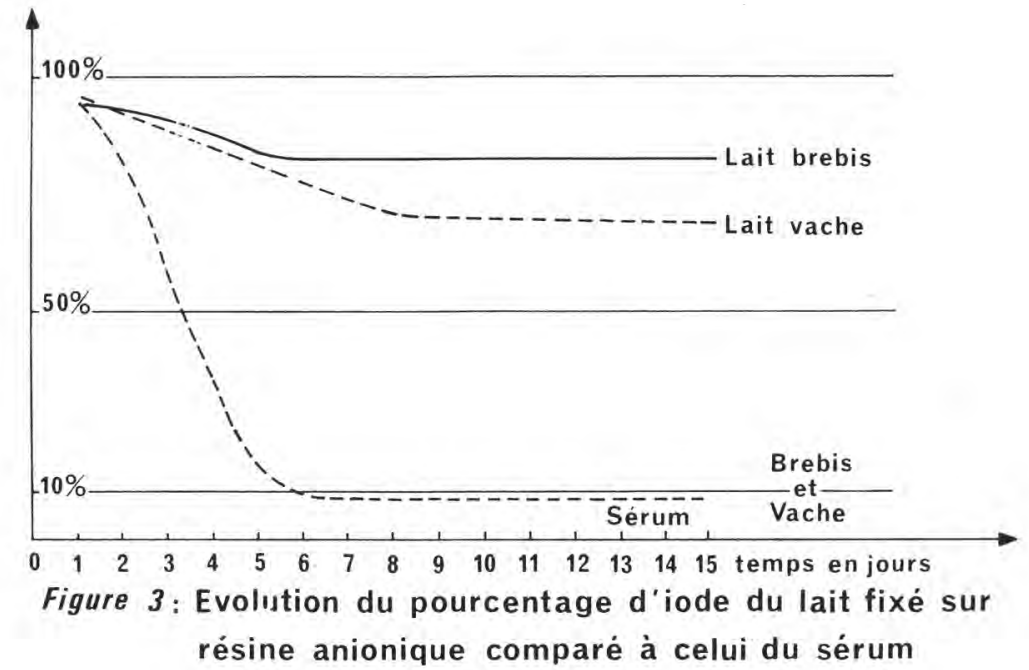

Chez une brebis réutilisée un an après, la fixation thyroïdienne est quasi-nulle et le pourcentage d'iode du lait fixé sur résine anionique est constant et égal à 98 p. 100.

Alors qu'un excès d'iode stable bloque la captation d'iodure par la thyroïde, il semble qu'au niveau de la mamelle, ce soit la capacité de fixation de l'iode sur les protéines du lait qui se bloque.

Il se pose donc le problème de savoir l'origine de cet iode lié aux protéines du lait.

La fraction ionique du sérum (10 p. 100 en moyenne) est capable, à elle seule, de fournir toute la quantité d'iode excrétée par le lait. En effet, la comparaison avec le rein rend plausible une telle hypothèse : chez la brebis le débit sanguin rénal est de $1200 \mathrm{l} / \mathrm{j}$ et l'excrétion urinaire moyenne est de 30 p. 100 alors que le débit mammaire est de $1450 \mathrm{l} / \mathrm{j}$ et l'excrétion par le lait de 35 p. 100 [10].

Mais deux faits nous incitent à penser que l'iode organique du lait peut provenir du pool organique sanguin.

1) L'iode iodure, sous l'influence d'oxydants ou d'enzymes existant dans le lait (peroxydases et oxydases) peut se fixer solidement aux protéines du lait selon un processus analogue à celui qui est 
réalisé dans la thyroïde et que l'on peut observer également in vitro (tab. 1).

\section{TABLEAU 1}

Pourcentage d'iode lié aux protéines du lait après action, in vitro, de différents produits sur du lait contaminé à l'iode 131 [27]

\begin{tabular}{|c|c|c|c|}
\hline \multirow[b]{2}{*}{, } & \multicolumn{2}{|c|}{ Vache } & \multirow{2}{*}{$\begin{array}{c}\text { Brebis } \\
\begin{array}{r}\text { Lait cru } \\
\text { (p. 100) }\end{array}\end{array}$} \\
\hline & $\begin{array}{l}\text { Lait cru } \\
\text { (p. 100) }\end{array}$ & $\begin{array}{l}\text { Lait bouilli } \\
\quad(\text { p. 100) }\end{array}$ & \\
\hline 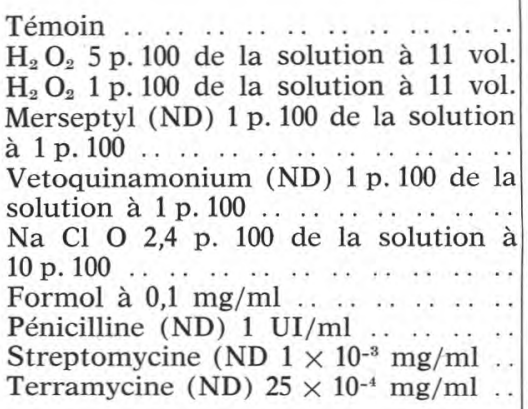 & $\begin{array}{r}2 \\
77 \\
43 \\
2 \\
7 \\
90 \\
38 \\
2 \\
2 \\
2\end{array}$ & $\begin{array}{r}1 \\
5 \\
9 \\
1 \\
1 \\
1 \\
91 \\
2 \\
1 \\
1 \\
1\end{array}$ & $\begin{array}{r}4 \\
-6 \\
4 \\
4 \\
4\end{array}$ \\
\hline
\end{tabular}

Mais une étude fine des répartitions de la radioactivité sur les protéines, après marquage in vitro et in vivo, nous montrent de grandes différences [27] (tab. 2).

$T A B L E A U 2$

\begin{tabular}{|c|c|c|c|c|}
\hline & \multicolumn{2}{|c|}{ In vitro } & \multicolumn{2}{|c|}{ In vivo } \\
\hline & $\begin{array}{l}\text { Vache } \\
\text { (p. } 100\end{array}$ & $\begin{array}{l}\text { Brebis } \\
(\text { p. 100) }\end{array}$ & $\begin{array}{l}\text { Vache } \\
\text { (p. 100) }\end{array}$ & $\begin{array}{l}\text { Brebis } \\
\text { (p. 100) }\end{array}$ \\
\hline Lait écrémé & & & & \\
\hline $\begin{array}{llllllllll}\text { Protéoses-peptones } & \ldots & \ldots & \ldots & \ldots & \ldots & \ldots \\
\text { Protéines } & \ldots & \ldots & \ldots & \ldots & \ldots & \ldots & \ldots & \ldots & \ldots\end{array}$ & $\begin{array}{l}23 \\
77\end{array}$ & $\begin{array}{l}26 \\
74\end{array}$ & $\begin{array}{l}77 \\
23\end{array}$ & $\begin{array}{l}62 \\
38\end{array}$ \\
\hline Lactosérum & & & & \\
\hline 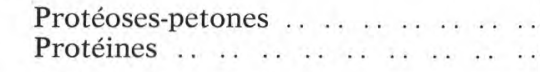 & $\begin{array}{l}71 \\
29\end{array}$ & $\begin{array}{l}85 \\
15\end{array}$ & $\begin{array}{l}90 \\
10\end{array}$ & $\begin{array}{l}80 \\
20\end{array}$ \\
\hline
\end{tabular}

On voit donc que dans le lait, les pourcentages d'iode fixés sur les protéoses-peptones et les autres protéines (caséine surtout) sont inversés.

2) Le pourcentage d'iode lié aux protéines du lait augmente dans le temps de façon assez parallèle à celui des P.B.I. du sérum, si l'on 
compare les courbes moyennes. Si la fixation était due uniquement à un phénomène physico-chimique ayant lieu dans la mamelle, on ne voit pas pourquoi le pourcentage n'en serait pas constant dès le premier jour.

Toutefois, il semble bien exister deux types de liaison de l'iode avec les protéines du lait :

- d'une part, une liaison chimique solide résistant aux résines anioniques, aux acidifications à $\mathrm{pH} 4,9$, au chauffage à $45^{\circ} \mathrm{C}$ pendant plusieurs heures [27] correspondant vraisemblablement à une halogénation des groupements tyrosiniques (éventuellement d'autres acides aminés cycliques tels que phénylalanine, tryptophane, histidine) de divers constituants des protéines de lait et en un lieu encore inconnu,

- d'autre part, une liaison plus labile, sans doute une simple adsorption qui rend compte des différences observées dans l'évaluation de l'iode lié par deux méthodes autres que l'échange d'ions :

a) par précipitation, on obtient un pourcentage toujours supérieur à celui déterminé par les résines, par exemple 5 à 10 p. 100 dans le précipité [18-27-28] à l'acide trichloracétique ou à l'hydroxyde de zinc et 100 p. 100 de fixation sous résine anionique,

b) par chromatographie d'exclusion-diffusion (passage du lait sur gel de Séphadex), on retrouve par exemple 78 p. 100 de la radioactivité sur les protéines contre 46 p. 100 après passage sur résine anionique ; dans ce cas, le pourcentage adsorbé peut être évalué à $78-46=32$ p. 100 .

Un autre phénomène, d'ordre physiologique, influe sur le pourcentage de cette liaison. Lorsque l'on compare la fraction liée obtenue dans le lait de traite rapprochée (toutes les $8 \mathrm{~h}$ ) ou de rétention (toutes les $32 \mathrm{~h}$ ), on voit [18] que le pourcentage non échangeable est plus élevé dans le lait provenant des quartiers de rétention. Selon Miller et Swanson, l'iode iodure passe dans la mamelle dans les deux sens, s'équilibrant avec la teneur en iodure du sang, tandis que l'iode lié reste dans la mamelle. Nous avons noté dans nos expériences pendant les 5 premiers jours après administration unique, que la fraction liée était le matin (traite de $8 \mathrm{~h}$ ) environ 5 p. 100 plus élevée que dans le lait du soir (traite à $17 \mathrm{~h}$ ); les intervalles de traites étaient de 15 à $9 \mathrm{~h}$.

En ce qui concerne une origine possible de la fraction liée aux protéines correspondant au premier type de liaison décrit ci-dessus à partir des P.B.I. du sérum, il faut rappeler qu'on admet avec Blaxter (1959) [2] que l'épithélium mammaire est imperméable aux hormones thyroïdiennes qui constituent la plus grande partie de l'iode organique sanguin chez l'individu normal [11-17].

Une administration continue de thyroxine, chez la vache, diminue le pourcentage d'iode lié dans le lait d'un facteur de 0,7. Par 
contre, la fraction liée du lait après administration unique de thyroxine (marquée cette fois) est 1,6 fois plus élevée qu'après du INa [21].

Il existe dans le sérum, une fraction organique non hormonale [17] dont la composition est mal connue (acides aminés iodés peptides iodés) et la teneur faible chez les euthyroïdiens. Il est possible qu'après une administration de radioiode, ce pourcentage augmente ; il reste à déterminer la dose seuil d'irradiation nécessaire.

Enfin, nous avons comparé pour deux séries d'expérimentations menées chez 4 brebis, l'effet de l'injection de MIT, DIT et INa marqués sur le pourcentage d'iode lié dans le lait (fig. 4).

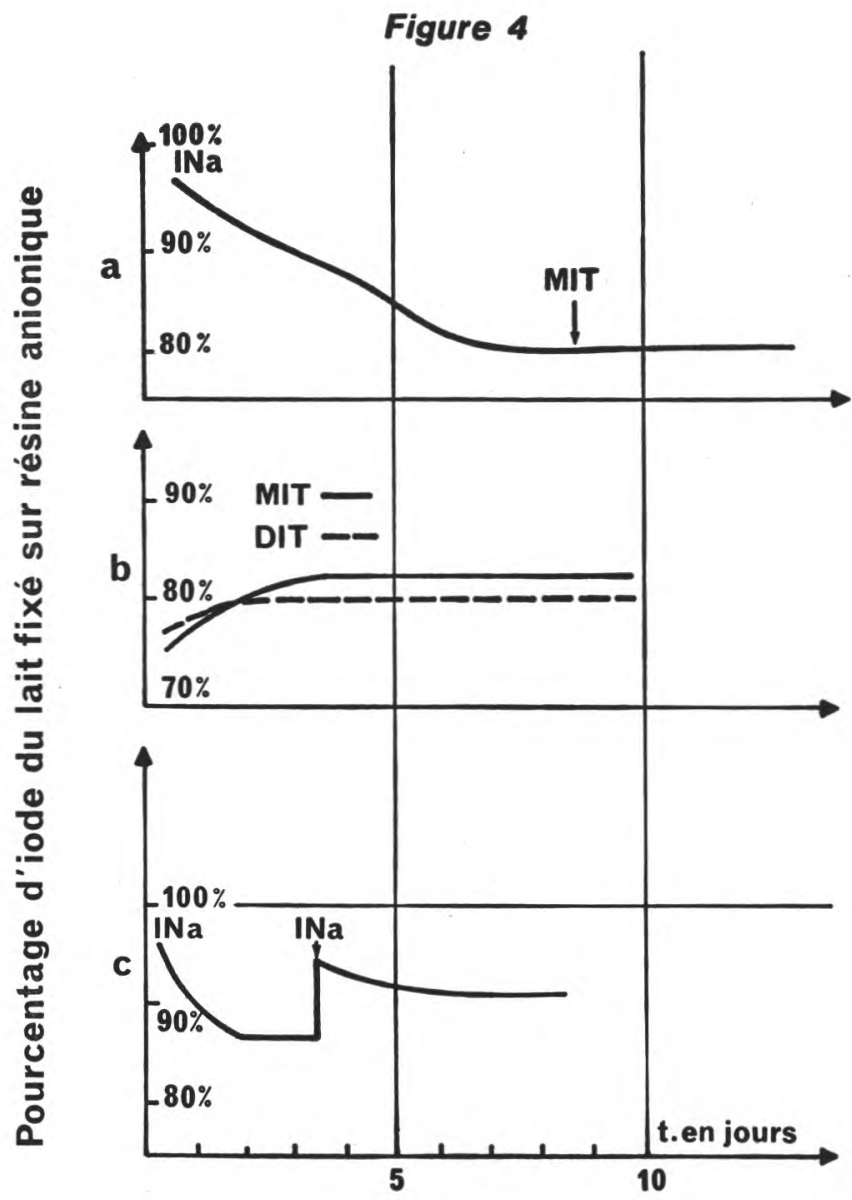

Lors d'une injection de MIT ou de DIT, le pourcentage lié part directement de 20 p. 100 et non de 0 à 5 p. 100 lors d'une injection d'INa (fig. 4 b). 
Lors d'une injection de MIT ou de DIT sur un animal ayant reçu du INa et dont la fraction liée du lait s'est stabilisée autour de 20 p. 100, on n'observe pas de variation nette de pourcentage (fig. 4 a).

$\mathrm{Si}$, dans les mêmes conditions d'expériences, on injecte de l'iodure, la fraction liée passe de 20 à 5 p. 100 puis revient à sa valeur initiale en quelques jours (fig. $4 \mathrm{c}$ ).

En comparant chez ces mêmes animaux utilisés successivement les quantités d'iode 131 excrétées par le lait après injection de ${ }^{131} \mathrm{INa}$ d'une part et de $\mathrm{M}^{131} \mathrm{IT}$ ou $\mathrm{D}^{131} \mathrm{IT}$ d'autre part, on trouve environ 40 à 45 p. 100 pour l'iodure et 50 à 55 p. 100 avec les acides aminés marqués.

Rappelons pour terminer que seuls, les acides aminés du sang participent à l'édification des protéines spécifiques du lait et non les peptides et que certains auteurs [1-26] ont noté la présence de MIT et DIT dans le sang d'homme et de rats normaux. Il reste à déterminer leur présence éventuelle chez les ruminants.

\section{V. - PASSAGE DE L'IODE 131 DU LAIT ENTIER A SES DERIVES [9]}

L'influence du mode de coagulation (présure, ferments lactiques) ne modifie pas de façon notable la répartition entre le fromage et le sérum ( 25 et 65 p. 100).

Le tableau 3 donne la différence entre laits contaminés in vivo et in vitro.

\section{TABLEAU 3}

Valeur du rapport $\frac{{ }^{131} \mathrm{I} \text { (dérivé) }}{{ }^{131} \mathrm{I} \text { (lait) }}$ après préparation du dérivé à partir de laits contaminés in vivo et in vitro

\begin{tabular}{|c|c|c|}
\hline Dérivé & $\begin{array}{l}\text { Contamination } \\
\text { in vivo }\end{array}$ & $\begin{array}{c}\text { Contamination } \\
\text { in vitro }\end{array}$ \\
\hline 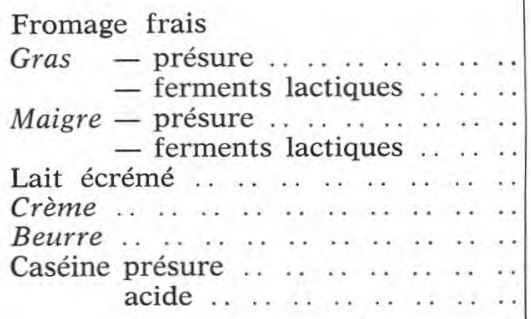 & $\begin{array}{l}3,03 \\
2,18 \\
2,78 \\
2,67 \\
0,99 \\
0,73 \\
0,36 \\
3,98 \\
3,59\end{array}$ & $\begin{array}{l}1,39 \\
1,51 \\
1,67 \\
1,37 \\
0,98 \\
0,78 \\
0,87 \\
1,81 \\
2,11\end{array}$ \\
\hline
\end{tabular}


TABLEAU 5. - Résultats en p. 100 de la dose contaminante

\begin{tabular}{|c|c|c|c|c|c|c|}
\hline & \multicolumn{2}{|c|}{$\mathrm{Eau}+\mathbf{M}^{131} \mathbf{I T}$} & \multicolumn{2}{|c|}{ Lait $+\mathrm{M}^{131} \mathrm{IT}$} & \multicolumn{2}{|c|}{ Lait $+\mathrm{D}^{131} \mathrm{IT}$} \\
\hline & Fixation & $\begin{array}{l}\text { Entraîné } \\
\text { par le } \\
\text { rinçage }\end{array}$ & Fixation & $\begin{array}{l}\text { Entraîné } \\
\text { par le } \\
\text { rinçage }\end{array}$ & Fixation & $\begin{array}{l}\text { Entraîné } \\
\text { par le } \\
\text { rinçage }\end{array}$ \\
\hline 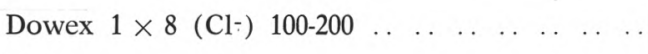 & 99,7 & 29,2 & 40,2 & 8,2 & 64,8 & 3,8 \\
\hline 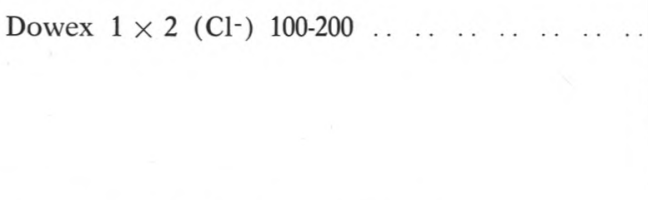 & 100 & 0,4 & 41,7 & $\begin{array}{c}18 \\
\text { dont } \\
\text { eau } 3,6 \\
+ \\
\text { sérum } \\
\text { salé } 14,4\end{array}$ & 63 & 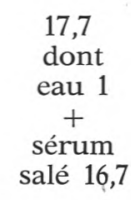 \\
\hline Dowex $50 \mathrm{~W} \times 2\left(\mathrm{NH}_{4}+\right) 100-200 \ldots \ldots \ldots \ldots$ & 96,5 & $\begin{array}{c}75,7 \\
\text { dont } \\
\text { eau } 29,3 \\
+ \\
\text { sérum } \\
\text { salé } 46,4\end{array}$ & & & & \\
\hline Dowex $50 \mathrm{~W} \times 4\left(\mathrm{NH}_{4}+\right) \quad 50-100 \ldots \ldots c c c$. & & & 30,2 & 8,6 & 15 & 4,8 \\
\hline
\end{tabular}




\section{APPENDICE}

A propos de la détermination des fractions ioniques et liées par les résines échangeuses, nous voudrions préciser quelques points qui peuvent donner une idée de la limite de la méthode.

- Lorsqu'on fait passer sur résine cationique (Dowex $50 \mathrm{~W} \times 4$ sous forme $\mathrm{NH}_{4}{ }^{+}$) du lait contaminé in vivo dans les mêmes conditions que ci-dessus, on obtient un pourcentage fixé de 10 p. 100 environ. Ces résines sont susceptibles de fixer les hormones thyroïdiennes et certains acides aminés iodés [17].

- Nous avons étudié (tab. 5) la fixation de la $\mathrm{M}^{\mathrm{r} 1 \mathrm{I}} \mathrm{IT}$ et de la $\mathrm{D}^{131} \mathrm{IT}$ sur les résines anioniques et cationiques dans le sérum physiologique et le lait, en rinçant les résines soit avec de l'eau distillée, soit avec du sérum salé à 9 p. 1000.

\section{METHODE}

- Sérum salé : $4 \mathrm{ml}$ de sérum salé à 9 p. 1000 sur $4 \mathrm{ml}$ de résine. Rinçage : 3 fois $4 \mathrm{ml}$ d'eau désionisée et 2 fois $4 \mathrm{ml}$ de sérum salé.

- Lait : Agitation de $50 \mathrm{ml}$ de lait marqué avec $3 \mathrm{ml}$ de résine pendant $20 \mathrm{mn}$. Filtration pour séparer la résine. Rinçage du filtre et de la résine par $50 \mathrm{ml}$ de sérum physiologique et $50 \mathrm{ml}$ d'eau désionisée.

Pour le lait contaminé avec de la $\mathrm{M}^{131} \mathrm{IT}$, le rendement de fixation sur résine, obtenu par agitation s'élève à 83 p. 100 de celui obtenu par passage sur colonne.

On note sur le tableau 5, la grande variabilité des pourcentages élués en fonction de la nature de l'éluant, ce qui doit rendre prudent dans l'interprétation des résultats obtenus avec les résines échangeuses d'ions dans ce type d'étude.

\section{Rés u m é}

Après un exposé rapide de leurs résultats personnels concernant les bilans d'excrétion de l'iode chez la brebis et chez la vache en lactation, les auteurs passent en revue les facteurs physico-chimiques, physio-pathologiques et pharmaco-dynamiques qui peuvent influer sur le passage de l'iode dans le lait.

Dans une seconde partie, les variations respectives des pourcentages d'iode ionique et d'iode lié aux protéines du lait sont étudiées en fonction des divers paramètres et différentes hypothèses relatives à l'origine de cette fraction organique sont proposées. 


\section{Su $\mathrm{m}$ m a ry}

After a short account of the results which they obtained concerning the iodine excretion balance in lactating ewes and cows, the authors review the physicochemical, physiopathological and pharmacodynamic factors which may influence the transfer of iodine to milk.

In a second part, the respective variations of the percentages of ionic and milk-protein-bound iodine are studied with respect to the different parameters and several assumptions concerning the origin of the organic fraction are put forward.

\section{Bibliographie}

[1] Beale (D) and Whitehead (J. K.) (1960). - A preliminary investigation of the levels of 3-monoiodotyrosine and 3,5-diiodotyrosine in human blood plasma using double isotope dilution technique. Clin. Chim. Acta, 150-152.

[2] Blaxter (K. L.), Reineke (E. P.), Crampton (E. W.) and Petersen (W. E.) (1949), - The role of thyroidal materials and of synthetic goitrogens in animal production and an appraisal of this practical use. J. Anim. Science, 8, 307.

[3] Block (R. J), Werner (S. C.), Mandl (R. H.), Row (V. V.) and Radichevich (I.) (1960). - The probable presence of diiodotyrosine and monoiodotyrosine in human serum. A discrepancy betwen the distribution of iode compounds when estimated by ${ }^{131} \mathrm{I}$ and by ${ }^{127} \mathrm{I}$. Arch. Biochem. Biophys., 88, 98-104.

[4] Comar (C. L.). (1968). - Studies on the secretion of iodine into the milk of thyroidectomized goats. NYO-2147-13, 53-55.

[5] Daburon (F.), Capelle (A.), Tricaud (Y.) et Nizza (P.) (1967). - Résultats expérimentaux relatifs au métabolisme de l'iode 131 ingéré chez la brebis adulte en lactation. Rapport C.E.A. - R. - 3107.

[6] Daburon (F.), Capelle (A.), Tricaud (Y.) et Nizza (P.) (1968). - Quelques aspects du métabolisme de l'iode chez les ruminants. Rev. Méd. Vét., 119 (4), 323.

[7] Garner (R. J.) and ScotT-Russel (R.) (1966), - Isotopes of iodine. Dans : Radioactivity and human Diet, chap. 14, Oxford. Pergamon Press.

[8] Jeanmaire (L.), Patti (F.), Daburon (M. L.), Gros (R.) et Bertrand (S.) (1966). - Essais relatifs au dosage de l'iode 131 dans le lait au moyen d'un échangeur d'ion anionique. Rapport C.E.A. - R - 2897.

[9] Kirchmann (R.), Adam (V.) et Van Puymbroeck (S.) (1966). - Radiocontamination des dérivés du lait de vache. Dans : Radioisotopes and radiation in Dairy Science and technology. I.A.E.A., Vienne.

[10] Kolb (E.) (1965). - Physiologie des animaux domestiques. Trad. par LABIE (Ch.), Paris. Vigot.

[11] Labadie (P.) (1967). - L'exploration fonctionnelle du corps thyroïde. Rev. du Prat., 17, (9 bis), 1386-1396.

[12] Lengemann (F. W.) (1970). - Metabolism of radioiode by lactating goats given iodine-131 for extended periods. J. Dairy Science, 53 (2), 165-170.

[13] Lengemann (F. W.) (1965). - The study of iodine secretion into the milk of dairy animals. Dans : Radioisotopes in animal nutrition and physiology, 203-220. I.A.E.A., Vienne.

[14] Lengemann (F. W.) (1965), - Factors affecting iodine concentration in bovin milk. J. Dairy Science, 48, 197-202. 
[15] Lengemann (F. W.) (1969). - Radioiodine in the milk of cows and goats after oral administration of radioiodate and radioiodide. Health Physics, 17, (4), 565-569.

[16] Lengemann (F. W) and Swanson (E. W.) (1957). - A study of the secretion of iodine in milk of dairy cows, using oral doses of ${ }^{131}$ I. J. Dairy Science, 40, (3), 216-224.

[17] Meyniel (G.), Blanquet (P.), Plagner et Jourde (M.) (1965). - L'étude des composés iodés du sang au cours de l'exploration fonctionnelle de la thyroïde par les isotopes radioactifs. Path. Biol., 13, (n $\left.{ }^{\circ} 19-20\right), 942-960$.

[18] Miller (J. K.) and Swanson (E. W.) (1963). - Some factors affecting iodine secretion in milk. J. Dairy Science, 46 (9), 927-932.

[19] Miller (J. K.), Moss (B. R.) and Swanson (E. W.) (1969). - Effects of ${ }^{131} \mathrm{I}$ thyroid irradiation domage, thyroxine and thiocyanate on radioiodine metabolism in dairy cows. J. Dairy Science, 52 (5), 677-683.

[20] Miller (J. K.) and Swanson (E. W.) (1967). - Performance and iodine metabolism of dairy cattle with iodine 131 irradiation. J. Dairy Science, 50 (1), 90-95.

[21] Miller (J. K.), Swanson (E. W.) and Cragle (R. G.) (1963). - Effect of feeding, thyroxine on secretion of ${ }^{131} \mathrm{I}$ in milk. J. Dairy Science, 46, 819.

[22] Morgan (A.) (1960). - Measurements of non-ionic iodine in the milk of dairy cows folloving oral administration of labelled sodium iodide. $J$. Dairy Science, 27, 399.

[23] Potter (G. D.) and Mc Intyre (D. R.) (1968). - In vitro analysis of the binding of ${ }^{131}$ I iodide to milk protein. J. Dairy Science, 51 (8), 1177-1181.

[24] Premachandra (B. N.) and Turner (C. W.) (1961). - Effect of mild hypertyroidism upon secretion of ${ }^{131} \mathrm{I}$ in milk of dairy cattle. J. Dairy Science, 44, 2035.

[25] REINEKE (E. P.) (1961). - Factors affecting the secretion of iodine 131 into milk of lactating goats. J. Dairy Science, 44, 937.

[26] RHodes (B. A.) and Wagner (H. N.) jr. (1966). - Are iodotyrosine normal constituents of plasma ? Nature, 210, 647-648.

[27] Romain (J.) (1970). - Contribution à l'étude de la fixation de l'iode radioactif sur les protéines des laits de vache et de brebis. Thèse Doct. Vét., Paris.

[28] Stroup (W. N.), Reyes (A. L.), Murthy (G. K.), Read (R. B.) and Dickerson (R.W.) (1968). - Combined process for removing radioactive iodine and strontium from milk by ion exchange. J. Dairy Science, 51 (9), 1500-1502.

[29] Swanson (E. W.), Miller (J. K.) and Cragle (R. G.) (1965). - Metabolism of different forms of iodine in milk given to calves by suckling and rumen infusion. J Dairy Science, 48 (7), 930-934.

[30] Tennessee University, Knoxville, Agricultural research program (1955). Radioisotopes in the investigations of the physiology of milk secretion. Progress report, ORO-145.

[31] Wright (W. E.), Christian (J. E.) and Andrews (F. N.) (1955). - The mammary elimination of radioiodine. J. Dairy Science, 38, 131-136. 